Br Heart J 1988;59:253-5

Case reports

\title{
Percutaneous coronary angioplasty in a cardiac transplant recipient
}

\author{
M D GAMMAGE, M F SHIU, T A H ENGLISH* \\ From the Department of Cardiovascular Medicine, University of Birmingham, East Birmingham and Queen \\ Elizabeth Hospitals, Birmingham and ${ }^{\star B r i t i s h ~ H e a r t ~ F o u n d a t i o n ~ H e a r t ~ T r a n s p l a n t ~ R e s e a r c h ~ U n i t, ~ P a p w o r t h ~}$ \\ Hospital, Cambridge
}

SUMMARY Two years after an orthotopic cardiac transplant a 28 year old man was found to have clinically significant stenosis of the right coronary artery at routine coronary angiography. This lesion was accompanied by a perfusion defect on exercise thallium scintigraphy.

Percutaneous transluminal coronary angioplasty of the lesion improved the angiographic appearance of the stenosis and reduced the exercise thallium perfusion defect.

The development of coronary artery disease in the transplanted heart is a cause for concern as it is usually asymptomatic and may progress rapidly, ${ }^{1}$ leading to silent myocardial infarction, cardiac failure, or sudden death. ${ }^{2}$ Retransplantation has been performed in cases in which coronary atherosclerosis had progressed to diffuse, extensive involvement of epicardial vessels. ${ }^{13}$ Early detection of coronary disease is therefore important and most centres practise annual angiography of the coronary vessels, usually after the first two years. Because the transplanted heart is denervated, anginal symptoms are absent even when there is severe disease. The most useful approach to management of early discrete lesions is uncertain.

We report a case in which a significant discrete stenosis of the right coronary artery was discovered two years after transplantation; this was treated by percutaneous transluminal coronary angioplasty with a good result.

\section{Case report}

A 26 year old man underwent orthotopic cardiac transplantation in November 1984 because of intractable congestive cardiac failure after a large anterior wall myocardial infarction caused by occlusion of the left anterior descending coronary artery. The other two vessels were normal. He was a non-smoker with a normal lipid profile, but he had a strong family history of coronary artery disease. The donor heart

Requests for reprints to Dr M D Gammage, Professorial Department of Medicine, Leicester Royal Infirmary, Infirmary Square, Leicester LE1 5WW. came from a 43 year old man with no history suggestive of coronary artery disease. There was also no evidence of coronary artery disease when the donor heart was inspected at the time of its removal. After transplantation, cyclosporin $B$ and azathioprine were given for immunosuppression and aspirin and dipyridamole were also given. The postoperative course was complicated only by a persistent nodal rhythm resulting in bradycardia (45-50 beats/ $\mathrm{min}$ ); this was, however, asymptomatic. A permanent, epicardial pacemaker (Medtronic Spectrax, VVI) had been fitted as a standard procedure at the time of transplant, and the device produced occasional episodes of ventricular paced rhythm. He was discharged home on the 35th postoperative day and returned to a normal active life. He experienced no clinical or histological rejection episodes in the next two years but at routine coronary angiography in November 1986 he was found to have an appreciable stenosis of the mid-right coronary artery (fig 1a). Exercise thallium scintigraphy showed a large defect in the lateral segment with moderate reperfusion in the resting images at four hours after exercise (fig 2a).

In view of these abnormalities, percutaneous transluminal coronary angioplasty of the right coronary artery was undertaken in February 1987. A femoral approach was used with the standard technique of dilatation with a steerable $3.0 \mathrm{~mm}$ balloon catheter and a Judkins' guiding catheter. There was no residual angiographic stenosis or complications after dilatation of the lesion (fig 1b). Repeat exercise thallium scintigraphy at six weeks after angioplasty showed a smaller lateral perfusion defect than demonstrated before, but there was no evidence of reperfusion at four hours after exercise (fig 2b). The 

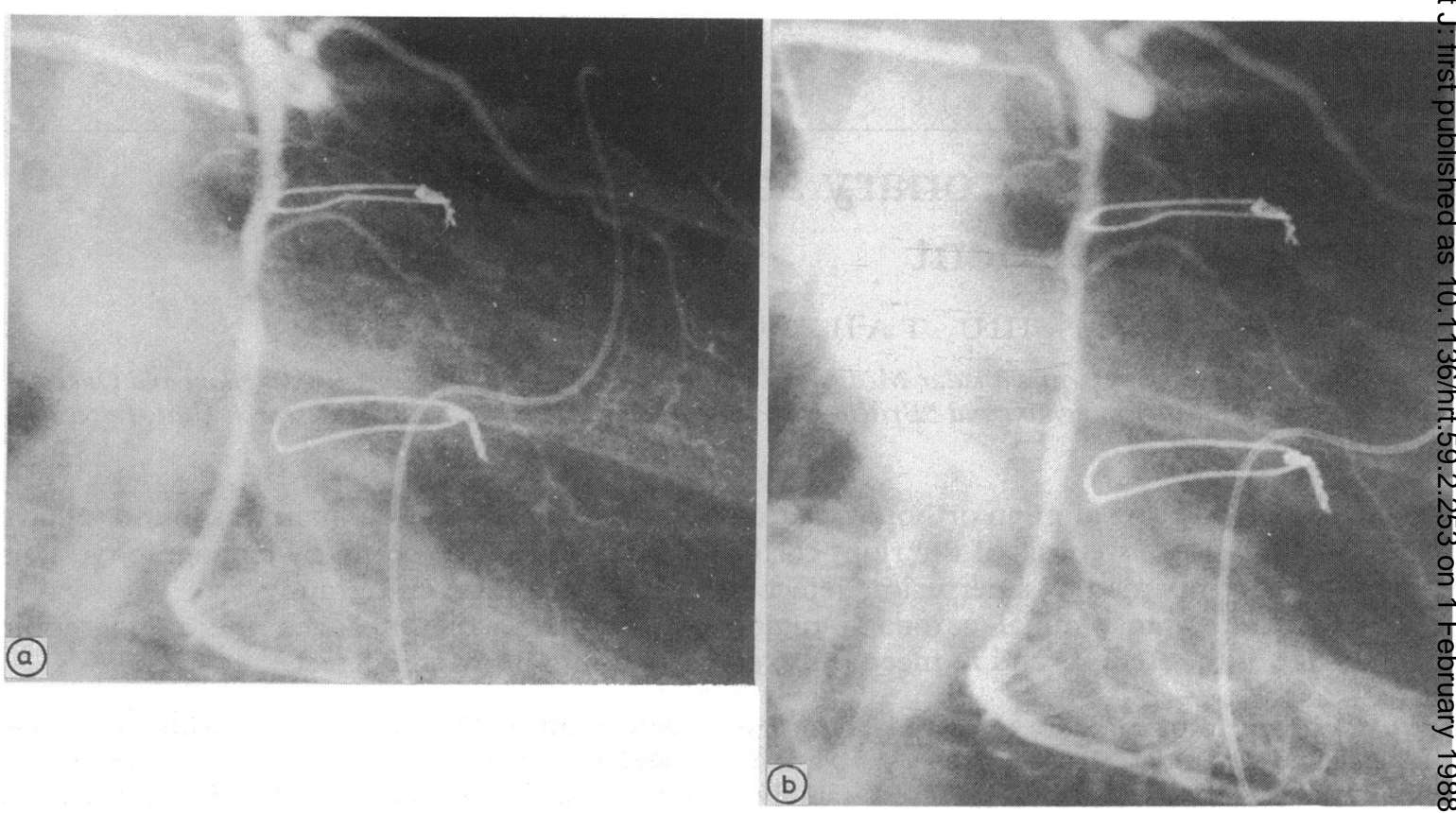

Fig 1 Angiogram of the right coronary artery (right anterior oblique view) showing (a) significant stenosis in the midsection and (b) the same view after angioplasty.
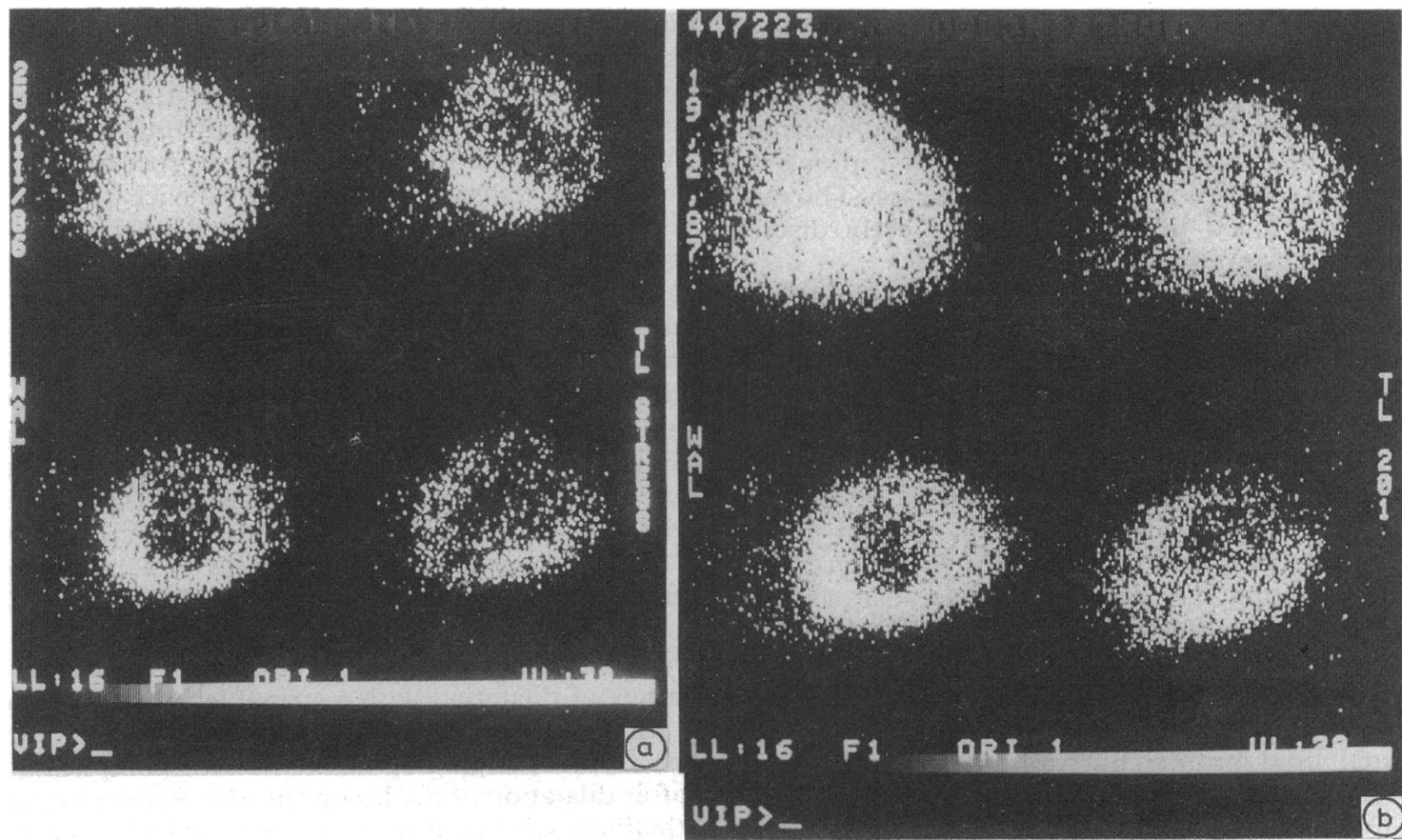

Fig 2 Four-view (anterior, $30^{\circ}$ left anterior oblique, $60^{\circ}$ left anterior oblique, left lateral) thallium-201 scintigram (stress images) showing (a) large defect in the lateral segment (top right) before angioplasty and the same views (b) six weeks after angioplasty showing some improvement in perfusion. 
patient remains well and continues to have a good exercise tolerance. Repeat coronary angiography at six months after angioplasty showed a patent right coronary artery with good remodelling and only minor residual stenosis.

\section{Discussion}

The aetiology of accelerated coronary occlusive disease in some transplanted hearts is imperfectly understood. It is likely that rejection is a feature, at least as an initiating factor, and both cytotoxic B-cell antibodies and hypercholesterolaemia have been implicated in the pathogenesis. ${ }^{4}$ Our own experience indicates that there is angiographic evidence of disease in $25 \%$ of patients five years after transplantation, whereas others have reported an incidence of $40 \%$ at five years. ${ }^{1}$. Whatever the true figure, coronary artery disease is undoubtedly the most important determinant of survival in the medium and long term after transplantation and if it is severe retransplantation may be required.

There are two reports of angioplasty in transplanted hearts in the United States. ${ }^{56}$ In both there was multivessel rather than single vessel disease. Our patient had an isolated lesion and, as the donor was a 43 year old man, it is possible that this was present in the donor organ at the time of transplantation. This cannot be disproved because the first angiogram was performed at two years. Initially it was our practice to perform angiography once a year from the first year onwards, but we no longer do this because the incidence of disease at one year was so low. Nevertheless, the lesion may have developed between transplantation and the angiogram at two years as, although accelerated coronary occlusive disease is often diffuse, we have found the appearance of segmental lesions after previously normal angiograms within this period.

The role of myocardial perfusion imaging with thallium-201 in the transplanted heart is less well defined than its role in "native" coronary artery disease. ${ }^{7}$ The single reported case presenting scintigraphic data $^{6}$ demonstrated an improvement of regional perfusion in the area of a previous perfusion defect after angioplasty of the stenosed vessel supplying the region, but other studies of thallium-201 imaging in the transplanted heart with diffuse small vessel disease have demonstrated a poor sensitivity in these circumstances. ${ }^{1}$

Angioplasty has now been shown to be technically possible in the transplanted heart with single or multiple vessel disease; the long term results in terms of patient and graft survival or vessel patency remain to be assessed.

We wish to thank Professor WA Littler for permission to report the case of a patient under his care. We also thank Dr Hywel Davies for his invaluable assistance in the preparation of this report.

\section{References}

1 Nitkin RS, Hunt SA, Schroeder JS. Accelerated atherosclerosis in a cardiac transplant patient. $\mathrm{J} \mathrm{Am} \mathrm{Coll}$ Cardiol 1985;6:243-5.

2 Hunt SA, Harrison DC, Stinson EB. Cardiac transplantation. In: Willerson JT, Sanders CA, eds. Clinical cardiology. New York: Grune and Stratton, 1977:613-23.

3 Watson DC, Reitz BA, Oyer PE, Stinson EB, Shumway NE. Sequential orthotopic heart transplantation in man. Transplantation 1980;30:401-3.

4 Hess ML, Hastillo A, Mohanakumar T, et al. Accelerated atherosclerosis in cardiac transplantation: role of cytotoxic B-cell antibodies and hyperlipidaemia. Circulation 1983;68(suppl II):II94-101.

5 Hastillo A, Cowley MJ, Vetrovec G, et al. Serial coronary angioplasty for atherosclerosis following heart transplantation. Heart Transplant 1985;4: $192-5$.

6 Wohlengarter D, Stevenson LW, Brunken R. Reversal of ischemic myocardial dysfunction by PTCA in a cardiac transplant patient. Am Heart J 1986;112: 837-9.

7 McKillop JH, Goris ML. Thallium-201 myocardial imaging in patients with previous cardiac transplantation. Clin Radiol 1981;32:447-9. 